\title{
Effects of vitamin D-induced supernatant of placental explants from preeclamptic women on oxidative stress and nitric oxide bioavailability in human umbilical vein endothelial cells
}

\author{
P.R. Nunes ${ }^{1 \oplus \bowtie}$, V.J. Gomes ${ }^{1 \oplus}$, V.C. Sandrim ${ }^{3 \oplus}$, J.C. Peraçoli ${ }^{1 \oplus}$, M.T.S. Peraçoli ${ }^{1 \oplus}$, and \\ M. Carlström ${ }^{2}$ \\ ${ }^{1}$ Departamento de Ginecologia e Obstetrícia, Faculdade de Medicina de Botucatu, \\ Universidade Estadual Paulista "Júlio de Mesquita Filho", Botucatu, SP, Brasil \\ ${ }^{2}$ Department of Physiology and Pharmacology, Karolinska Institutet, Stockholm, Sweden \\ ${ }^{3}$ Departamento de Biofísica e Farmacologia, Instituto de Biociências, Universidade Estadual Paulista \\ "Júlio de Mesquita Filho", Botucatu, SP, Brasil
}

\begin{abstract}
The study evaluated the effect of the supernatant of placental explants from preeclamptic (PE) and normotensive (NT) pregnant women after tissue treatment with or without vitamin $D(V D)$ on oxidative stress and nitric oxide (NO) bioavailability in human umbilical vein endothelial cells (HUVEC). Placental explants were prepared from eight NT and eight PE women, and supernatants were obtained after incubation with or without hydrogen peroxide $\left(\mathrm{H}_{2} \mathrm{O}_{2}\right)$ and/or VD. HUVEC were cultured for $24 \mathrm{~h}$ with supernatants, and the following parameters were analyzed in HUVEC cultures: $\mathrm{NO}$, nitrate $\left(\mathrm{NO}_{3}{ }^{-}\right)$, and nitrite $\left(\mathrm{NO}_{2}^{-}\right)$ levels, lipid peroxidation, and intracellular reactive oxygen species (ROS). Results showed that the production of $\mathrm{NO}_{3}^{-}, \mathrm{NO}_{2}^{-}$, malondialdehyde (MDA), and ROS were significantly higher in HUVEC treated with explant supernatant from PE compared to NT pregnant women, while the supernatant of PE explants treated with VD led to a decrease in these parameters. A significantly high production of NO was detected in HUVEC cultured with control supernatant of NT group, and in cultures treated with supernatant of PE explants treated with VD. Taken together, these results demonstrated that cultures of placental explants from $\mathrm{PE}$ women with VD treatment generated a supernatant that decreased oxidative stress and increased the bioavailability of NO in endothelial cells.
\end{abstract}

Key words: Oxidative stress; Placental explants; Preeclampsia; Vitamin D

\section{Introduction}

Preeclampsia (PE) is a specific human syndrome of pregnancy characterized as the main cause of morbidity, mortality, and preterm birth, affecting as many as $10 \%$ of all pregnancies. Clinical diagnosis is performed from the twentieth week of pregnancy or in the first days after delivery, based on the development of hypertension with or without proteinuria (1) in addition to maternal manifestations such as thrombocytopenia, impaired liver function, renal insufficiency, pulmonary edema, and new-onset cerebral or visual disturbances (2).

Nitric oxide (NO) is a key signaling molecule in the cardiovascular system, controlling vascular tone, neurotransmission, redox signaling, cellular respiration, and host defense (3). This molecule participates actively in the pregnancy processes such as trophoblast invasion and placental development, representing the main vasodilator in the placenta (4). Disturbances in the NO system, coupled with oxidative stress, contribute to vascular dysfunction in preeclamptic women (5). Oxidative stress, characterized by excessive formation of reactive oxygen species (ROS), can impair endothelial nitric oxide synthase (eNOS) function (6) and consequently decrease bioavailability of NO. Together with the excessive production of ROS, the placenta from preeclamptic women shows an intense inflammatory process. ROS are involved in the injuries signaling to the immune system (7) and can orchestrate the inflammatory response by the release of hydrogen peroxide $\left(\mathrm{H}_{2} \mathrm{O}_{2}\right)$

Correspondence: P.R. Nunes: <priscilarezeck@gmail.com> 
from damaged tissues leading to the recruitment of leukocytes to the lesion site (8).

Vitamin D (VD) has several effects on the organism, modulating cardiovascular and immune cell functions. Concerning the innate and adaptive immune system, it can establish a more tolerogenic immune status, by its regulatory activities on the inflammatory response. During normal pregnancy, VD is produced by placental trophoblast cells and human decidua and is responsible for anti-inflammatory effects in various organs, including the placenta (9).

This hormone plays an important role in the implantation, placentation, and maintenance of healthy gestations. During human pregnancy, the conversion of inactive $25(\mathrm{OH})-\mathrm{D}$ to the active form $1.25(\mathrm{OH}) 2-\mathrm{D}$ is increased in the placenta demonstrating that this tissue and decidua are important in the bioactivation of VD (10). Recent studies have reported VD deficiency in pregnant women with PE $(11,12)$ and others have shown an association between deficiency and the risk of developing $P E$, suggesting that supplementation may modulate the immune response in this pathology (13).

Recently, we demonstrated the in vitro activation of NLRP3 inflammasome in placental explants from normotensive (NT) pregnant women as a consequence of the $\mathrm{H}_{2} \mathrm{O}_{2}$-induced cellular stress, which is initiated by ROS release, as well as increased gene expression of inflammatory cytokines (14). Furthermore, the increase of ROS formation could decrease the bioavailability of $\mathrm{NO}$, since some researchers have suggested that $P E$ should be characterized by a disruption of vascular dilation mediated by $\mathrm{NO}$ and disturbed by ROS (15). Therefore, the use of immunomodulatory substances like VD for the in vitro treatment of explants may lead to a better understanding of the systemic inflammation in PE and possibly propose alternative ways to treat this syndrome.

Considering that the placenta of women with PE shows oxidative stress, exacerbated inflammation, and NO system imbalance, this study aimed to evaluate the effect of the supernatant of placental explants from PE and NT pregnant women treated with or without VD on oxidative stress and NO bioavailability in human umbilical vein endothelial cells (HUVEC). We intended to observe whether the employment of substances with an immunomodulatory effect such as VD on placental tissue can be used to reduce oxidative stress in endothelial cells.

\section{Material and Methods}

The methodology employed in this study is demonstrated in a schematic figure (Supplementary Figure S1).

\section{Study population and ethics statement}

Placentas and blood from 8 NT and 8 PE pregnant women were collected. These pregnant women were admitted to the Obstetrics Unit of Botucatu Medical
School, Sao Paulo State University, Botucatu, SP, Brazil, between November 2017 and August 2018. Gestational age was calculated from the last menstrual period and confirmed by ultrasound dating. A pregnant woman was considered preeclamptic when, without a history of hypertension, she developed hypertension $(\geqslant 140 / 90$ $\mathrm{mmHg}$ ) associated or not with proteinuria ( $\geqslant 300 \mathrm{mg}$ in 24-h urine) after the 20th week of gestation (1). NT pregnant women did not present a personal history of hypertensive disorders before or during pregnancy. Proteinuria in 24-h urine was analyzed by a colorimetric method, the Technicon RA-XT automation system (Asinteg, Argentina), and uric acid was assessed by uric acid enzymatic Trinder (Biotrol Diagnostic, India) in the Clinical Laboratory of Botucatu Medical School.

Exclusion criteria included chronic hypertension, multiple gestations, prior preeclampsia, illicit drug use, and preexisting medical conditions such as diabetes, cancer, acute infectious disease, and cardiovascular, autoimmune, renal, and hepatic diseases. The study was approved by the Ethics Committee of the Botucatu Medical School, and written informed consent was obtained from all women involved in the study (Protocol number: 3.383.792). This work was carried out under the Code of Ethics of the World Medical Association (Declaration of Helsinki). Also, all mandatory laboratory health and safety procedures were complied with in the course of conducting any experimental work reported in this paper and all experiments were performed following relevant guidelines and regulations.

\section{Collection of placental tissue}

Placentas were collected at delivery by elective cesarean section. Immediately after delivery, all placentas considered for the study were examined macroscopically and processed within 10 min. Fragments of approximately $5 \times 5 \mathrm{~cm}$ were immediately removed from the central region of the placenta, constituting samples in contact with the maternal side (basal plate). After this initial collection, smaller fragments were washed in phosphate buffered saline (PBS) and separated from the decidual layer that is normally adhered to the basal plate. The terminal portions of the villi were observed in PBS (the villi were seen floating freely in the liquid) and were dissected into small sections to constitute explants.

\section{Culture of placental explants with hydrogen peroxide and vitamin D}

Placental tissue was obtained according to a previous study (14). Briefly, a total of $11 \mathrm{mg}$ of human villous tissue was cultured in RPMI 1640 culture medium (Thermo Fisher, USA) supplemented with $2 \mathrm{mM}$ L-glutamine (Sigma-Aldrich, USA), $40 \mathrm{mg} / \mathrm{mL}$ antibiotic/antimycotic (Sigma-Aldrich), and $10 \%$ fetal bovine serum (Gibco BRL Life Technologies, The Netherlands) in 24-well plates (SPL Life Sciences, Korea) during $24 \mathrm{~h}$ for stabilization. After stabilization, placental 
explants from PE and NT pregnant women were cultured for $24 \mathrm{~h}$ with or without $\mathrm{H}_{2} \mathrm{O}_{2}(100 \mu \mathrm{M})$ (Sigma-Aldrich) as a stimulus for oxidative stress induction, and with or without VD (100 nM) (Sigma-Aldrich), considered an immunomodulator. Placental explant supernatants obtained were stored at $-80^{\circ} \mathrm{C}$ for later experiments and analyses.

\section{Cytokine determinations}

The concentrations of interleukin (IL)-1 $\beta$, tumor necrosis factor (TNF)- $\alpha$, and IL-18 in supernatants of placental explants from NT and PE women, obtained after treatment with or without $\mathrm{H}_{2} \mathrm{O}_{2}$ and $V D$, were determined by Quantikine ELISA kits (R\&D Systems, USA) according to the manufacturer's instructions. Assay sensitivity limits were $1.0 \mathrm{pg} / \mathrm{mL}$ for IL-1 $\beta, 1.6 \mathrm{pg} / \mathrm{mL}$ for TNF- $\alpha$, and 5.15 $\mathrm{pg} / \mathrm{mL}$ for IL-18.

Vitamin D and vitamin D receptor (VDR) determination Blood sampling and vitamin D determination. Peripheral blood $(10 \mathrm{~mL})$ was obtained by venipuncture from the antecubital vein of $8 \mathrm{PE}$ women at the time of PE diagnosis and of $8 \mathrm{NT}$ pregnant women at the time they were matched to gestational age with PE women. Blood was collected into plastic tubes containing 5\% EDTA. After blood centrifugation at $4^{\circ} \mathrm{C}$ for $10 \mathrm{~min}$ at $1,200 \mathrm{~g}$, the plasma fraction was removed and aliquots were stored at $-80^{\circ} \mathrm{C}$ until vitamin $\mathrm{D}$ determination.

Vitamin D, 25(OH)D, was determined by the automated chemiluminescence microparticle immunoassay (CMIA) with an Architect 25-OH Vitamin D assay kit, by the Architect ${ }^{\circledR}$ i2000 analyzer (Abbott ${ }^{\mathbb{R}}$, USA ). The analytical sensitivity was $1.9 \mathrm{ng} / \mathrm{mL}$ and the coefficient of variation within and between assays was $<10 \%$, as described in the kit. The reference range was $0.0-160.0 \mathrm{ng} / \mathrm{mL}$, according to the method. Values $\geqslant 30 \mathrm{ng} / \mathrm{mL}$ were considered sufficient, from 21 to $29 \mathrm{ng} / \mathrm{mL}$ insufficient, and $<20 \mathrm{ng} / \mathrm{mL}$ deficient (16).

\section{VDR gene expression}

Placental explants from $8 \mathrm{PE}$ and 8 NT pregnant women were employed to determine the expression of VDR at the transcriptional level. Total RNA was extracted from the placental explants after culture using the Total RNA Purification Kit (Norgen Biotek Corp., Canada) following the manufacturer's protocol, and the reverse transcription-coupled polymerase chain reaction (qPCR) was performed as described previously (14). Briefly, isolated RNA was treated with DNAse I Amp Grade (Invitrogen, USA). Subsequently, the synthesis of complementary DNA (cDNA) was conducted using ImProm-II TM Reverse Transcription System, according to the manufacturer's protocol (Promega, USA). The qPCR was performed using RT GoTaq-qPCR Master Mix (Promega, USA) and the variants of the studied targets were aligned in the MEGA 5.1 program (www.megasoft ware.net) and each primer was subsequently selected by the software Primer-BLAST (NIH, USA). Primers located in exon-exon junctions guarantee the purity of the reaction, namely the absence of any genomic DNA that may contaminate it. The VDR primer sequence used in this study was (590)TGGAGACTTTGACCGGAACG(609) (704)GCTTCGCCTGAAGAAGCCT(686). Each reaction was set in duplicate and the conditions for the qPCR were as follows: initial denaturation at $96^{\circ} \mathrm{C}$ for $2 \mathrm{~min}$ and then 40 cycles at $95^{\circ} \mathrm{C}$ for $15 \mathrm{~s}$ and $60^{\circ} \mathrm{C}$ for $60 \mathrm{~s}$, followed by a melting curve. Expression values of the analyzed transcripts were normalized to that of the enzymeencoding glyceraldehyde-3-phosphate dehydrogenase gene (GAPDH) as follows: (684)CGTGGAAGGACTCAT GACCA(703) (801)GGCAGGGATGATGTTCTGGA(782). The calculation of the differential expression of selected genes was carried out by the data processing method compared with a standard curve (17). To analyze the relative expression, after the analysis of gene expression, we chose an RNA sample obtained from each group, which received a relative value of 100 . All other samples received values for that sample.

\section{HUVEC culture and incubation with supernatant of placental explants}

HUVEC (Lonza, Switzerland) were acquired with a certification proving that the cells were from the designated type. All experiments were performed using cells in the sixth passage and in quintuplicates. HUVEC were cultured until reaching $80 \%$ confluence and then incubated for $24 \mathrm{~h}$ in medium 199 (Gibco BRL Life Technologies) with $20 \%$ (v/v) supernatant pool from placental explants of PE women, and from NT explants previously cultured with or without $\mathrm{H}_{2} \mathrm{O}_{2}$ and/or VD as described above. The cells and HUVEC media were used to perform the assays. PrestoBlue ${ }^{\mathbb{R}}$ Cell Viability Reagent (Invitrogen) was employed to determine whether the supernatants pre-treated with $\mathrm{H}_{2} \mathrm{O}_{2}$ and/or VD were harmful to HUVEC showing cell viability.

\section{Measurement of nitrate, nitrite, and NO species}

Nitrate and nitrite levels produced by HUVEC after placental explant supernatant incubation for $24 \mathrm{~h}$ were assessed in HUVEC supernatant using a high-performance liquid chromatography (HPLC) system (ENO-20; Eicom, USA) as previously described (18). The ENO-20's high sensitivity and specificity were accomplished with the combination of a diazo coupling method and chromatography. The level of the diazo compound was measured by absorbance at $540 \mathrm{~nm}$ using a Spectramax iD3 multimode microplate reader (Hidex, Lablogic Systems, UK).

A total of $5 \times 10^{4}$ HUVEC per well were plated onto a black 96-well plate (Sigma-Aldrich). After $24 \mathrm{~h}$ of incubation with placental explant supernatants, the cells were washed with $95 \mu \mathrm{L}$ of PBS and incubated for $30 \mathrm{~min}$ at $37^{\circ} \mathrm{C}$. After this period, cells were loaded with $5 \mu \mathrm{L}$ of DAF-FM $^{\mathrm{TM}}(10 \mu \mathrm{M})$ (Sigma-Aldrich) and read for $60 \mathrm{~min}$ in 
$37^{\circ} \mathrm{C}$ using Spectramax iD3. The fluorescence signal was measured in a microplate reader (excitation $495 \mathrm{~nm}$, emission $535 \mathrm{~nm}$ ) and is reported as arbitrary units.

\section{Measurement of lipid peroxidation - TBARS}

Levels of lipid peroxidation in the supernatant of HUVEC cultured with pre-treated placental explants supernatant for $24 \mathrm{~h}$ were measured by thiobarbituric acid reactive substances (TBARS). An aliquot of $100 \mu \mathrm{L}$ of supernatant was mixed with $200 \mu \mathrm{L}$ of $10 \%$ cold trichloroacetic acid (TCA, Sigma-Aldrich), before being placed on ice for $15 \mathrm{~min}$ to precipitate protein. During this time, standards were prepared in a serial dilution with 1,1,1,3-tetramethoxypropane (TMP, $\mathrm{C}_{7} \mathrm{H}_{16} \mathrm{O}_{4}, 133.75 \mathrm{mM}$, Sigma-Aldrich). Then, the samples with TCA were centrifuged at $2,200 \mathrm{~g}$ for $15 \mathrm{~min}$ at $4^{\circ} \mathrm{C}$. After centrifugation, $200 \mu \mathrm{L}$ of the supernatants and standards were placed in cryotubes with an equal volume of $0.67 \%$ thiobarbituric acid (TBA, Sigma-Aldrich) and boiled at $95^{\circ} \mathrm{C}$ for $50 \mathrm{~min}$. Finally, the tubes were placed on ice for $3 \mathrm{~min}$. Standards and samples were placed on a 96-well plate and the absorbance was measured at $532 \mathrm{~nm}$ (Spectramax iD3) and the TBARS values were calculated using the malondialdehyde (MDA) standard curve. The values are reported as nanomoles of MDA per $\mathrm{mL}$.

\section{Levels of intracellular ROS}

Intracellular ROS was quantified in the supernatant of HUVEC cultured with pre-treated placental explants supernatant using 2'-7'-dichlorodihydrofluorescein diacetate (DCFH-DA; Sigma-Aldrich) by fluorescence with $2 \mathrm{~V}, 7 \mathrm{~V}$-dichlorofluorescein diacetate. Tert-butyl hydroperoxide (tBHP) at $1000 \mu \mathrm{M}$ was added $2 \mathrm{~h}$ before reading as a positive control. After $24 \mathrm{~h}$ of treatment with placental explant supernatants, HUVEC were incubated with $100 \mu \mathrm{M}$ DCFH-DA diluted in dimethyl sulfoxide (DMSO) for $30 \mathrm{~min}$ at $37^{\circ} \mathrm{C}$. Then, cells were washed with PBS $(\mathrm{pH} \mathrm{7.4)}$ and the relative levels of fluorescence were quantified in a spectrophotofluorimeter (Hitachi F-4500, Japan, $485 \mathrm{~nm}$ excitation and $520 \mathrm{~nm}$ emission). The measured fluorescence values are reported as fluorescence intensity.

\section{Statistical analysis}

Comparisons between groups were assessed by nonparametric tests (Mann-Whitney $U$ test) and parametric analysis of variance (ANOVA) followed by Bonferroni's multiple comparison test. Results were evaluated using the statistical program PRISM (Graph Prism, version 6.01, GraphPad, USA) and statistical significance was accepted at $\mathrm{P}<0.05$.

\section{Results}

Clinical and laboratory characteristics showed worse clinical outcomes in PE women

The differences between clinical and laboratory data of PE and NT pregnant women are reported in Table 1. There was no statistical difference in age between the groups. However, gestational age was lower in women with PE compared to NT. As expected, PE had worse clinical outcomes compared to NT pregnant women, and were associated with hypertension, hyperuricemia, and proteinuria.

\section{VD and VDR were lower in plasma and placental explants from PE women}

Figure 1A shows vitamin D $25(\mathrm{OH}) \mathrm{D}$ levels in the plasma of $\mathrm{PE}$ and NT pregnant women. Figure $1 \mathrm{~B}$ presents VDR gene expression in PE and NT placental explants. Both the VD levels and the receptor's gene expression were significantly lower in pregnant women with PE.

\section{Inflammatory cytokines were lower in supernatant from explants treated with VD}

Figure 2 shows the concentrations of cytokines in supernatant of placental explants from PE and NT pregnant women cultured in the absence or presence of $\mathrm{H}_{2} \mathrm{O}_{2}$ and VD. Protein expression of IL-1 $\beta$, TNF- $\alpha$, and IL-18 was significantly higher in supernatants from control cultures of PE compared to NT pregnant women. In the $\mathrm{PE}$ group, treatment with VD showed decreased expression of IL-1 $\beta$, TNF- $\alpha$, and IL-18 compared to control explants supernatant. The $\mathrm{H}_{2} \mathrm{O}_{2}+\mathrm{VD}$ treatment showed lower protein expression of IL-1 $\beta$, TNF- $\alpha$, and IL-18 compared to $\mathrm{H}_{2} \mathrm{O}_{2}$ treatment in cultures of NT and PE explants.

Table 1. Characteristics of the pregnant women.

\begin{tabular}{lcc}
\hline Variable & Normotensive $(\mathrm{n}=8)$ & Preeclampsia $(\mathrm{n}=8)$ \\
\hline Age (years) & $29(18-41)$ & $28(16-39)$ \\
Gestational age (weeks) & $40(36-41)$ & $34(30-39)^{\star}$ \\
Systolic blood pressure $(\mathrm{mmHg})$ & $120(110-120)$ & $155(140-70)^{\star}$ \\
Diastolic blood pressure $(\mathrm{mmHg})$ & $70(60-80)$ & $100(90-110)^{\star}$ \\
Uric acid $(\mathrm{mg} / \mathrm{dL})$ & $3.2(2.3-4.7)$ & $5.7(3.7-6.9)^{\star}$ \\
Proteinuria $(\mathrm{mg} / 24 \mathrm{~h})$ & $<300$ & $730(300-7540)^{*}$ \\
\hline
\end{tabular}

Data are reported as median (minimum-maximum). ${ }^{*} \mathrm{P}<0.05$ vs normotensive (Mann-Whitney U test). 

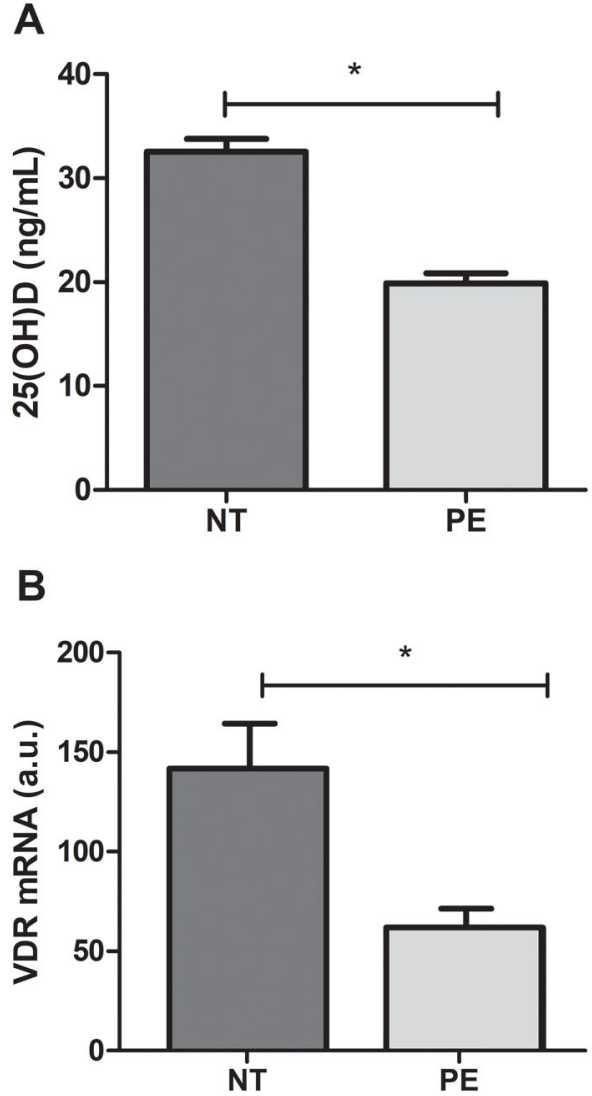

Figure 1. Levels of vitamin $25(\mathrm{OH}) \mathrm{D}$ in plasma (A) and vitamin $\mathrm{D}$ receptor (VDR) gene expression (B) in the placental explants from preeclamptic $(\mathrm{PE})(\mathrm{n}=8)$ and normotensive $(\mathrm{NT})$ pregnant women $(n=8)$. Data are reported as means $\pm S D$. ${ }^{*} P<0.05$ (Mann-Whitney $U$ test). a.u.: arbitrary units.

\section{Cell viability of HUVEC when treated with supernatants}

After performing the dosages of VD and cytokines, the supernatant of PE and NT explants was used to treat the cultures of HUVEC. Before carrying out the culture, a viability test was performed to observe if the supernatants were harmful to the cells. HUVEC viability after 24-h culture with placental explant supernatant from $P E$ and NT women pre-treated with $\mathrm{H}_{2} \mathrm{O}_{2}, \mathrm{H}_{2} \mathrm{O}_{2}+\mathrm{VD}$, and VD is presented in Supplementary Figure $\mathrm{S} 2$. No significant differences were found between treatments regarding cell viability.

Levels of nitrite and nitrate were significantly lower in HUVEC treated with supernatant of PE explants cultured with VD

Figure 3 shows nitrite and nitrate concentrations in supernatant of HUVEC after culture with supernatants of
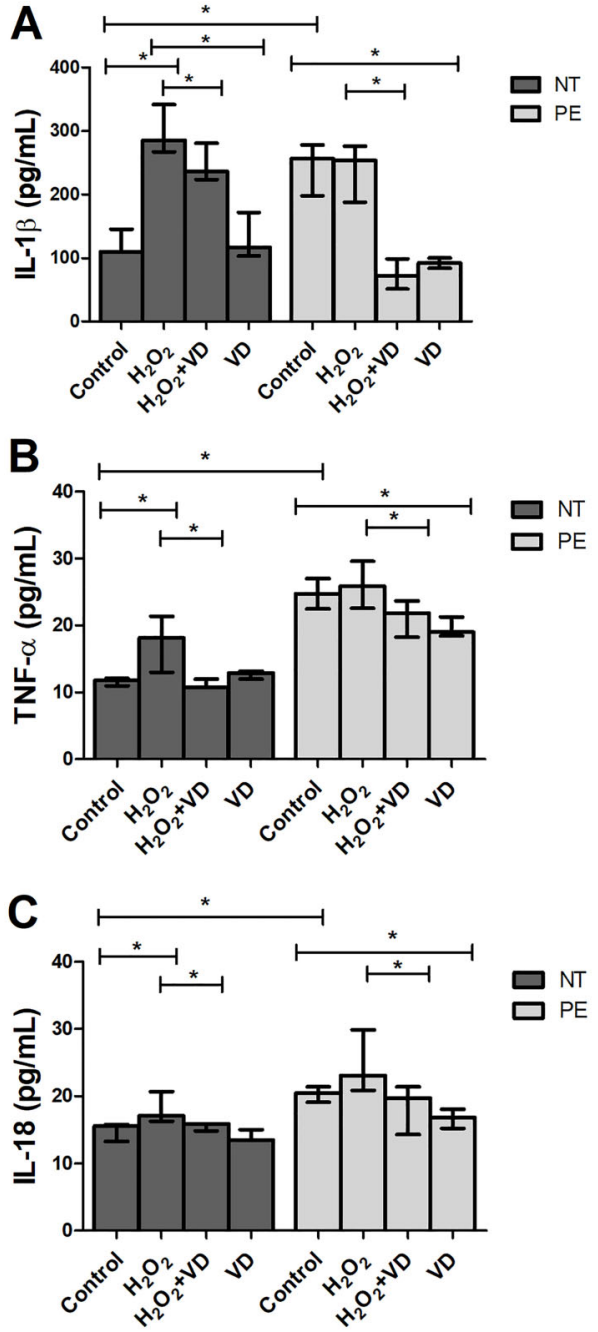

Figure 2. Concentration of cytokines in supernatant of placental explants from preeclamptic (PE) $(n=8)$ and normotensive (NT) pregnant women $(n=8)$ under different treatments. Data are reported as median with range. ${ }^{*} \mathrm{P}<0.05$ (Mann-Whitney $\mathrm{U}$ test). IL-1 $\beta$ : interleukin-1 beta; TNF- $\alpha$ : tumor necrosis factor-alpha; IL-18: interleukin-18; VD: vitamin D.

PE and NT placental explants pre-treated with $\mathrm{H}_{2} \mathrm{O}_{2}$, $\mathrm{H}_{2} \mathrm{O}_{2}+V D$, and VD. Levels of nitrite and nitrate were significantly higher in HUVEC cultures treated with PE control supernatant compared to NT control. Similarly, we observed higher levels of nitrite and nitrate in HUVEC cultures submitted to treatment with supernatant of placental explants from NT women treated with $\mathrm{H}_{2} \mathrm{O}_{2}$ compared to control supernatant. On the other hand, treatment of HUVEC with supernatant of PE explants cultured with VD led to a decrease in nitrite and nitrate levels compared to control supernatant, and also in the 
treatment with supernatant of placental explant of NT women cultured with $\mathrm{H}_{2} \mathrm{O}_{2}+\mathrm{VD}$ compared to only $\mathrm{H}_{2} \mathrm{O}_{2}$.
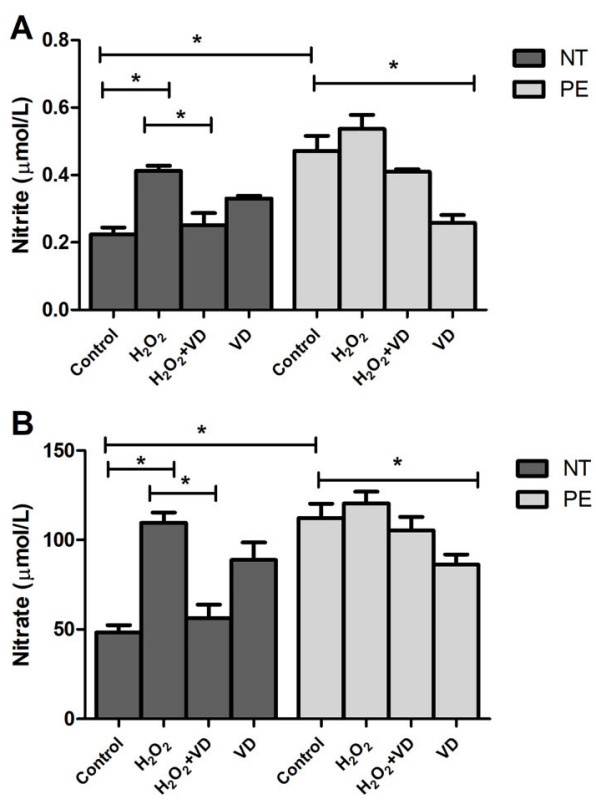

Figure 3. Levels of nitrite (A) and nitrate (B) in the supernatant of human umbilical vein endothelial cells treated with supernatant of placental explants from preeclamptic $(P E)(n=8)$ and normotensive (NT) pregnant women $(n=8)$, cultured in the absence (Control) or presence of $\mathrm{H}_{2} \mathrm{O}_{2}, \mathrm{H}_{2} \mathrm{O}_{2}+\mathrm{VD}$, and VD. Data are reported as means $\pm \mathrm{SD}$ for one independent experiment, performed in quintuplicates. ${ }^{*} \mathrm{P}<0.05$ (ANOVA followed by Bonferroni's multiple comparison test). VD: vitamin D.

\section{HUVEC treated with NT supernatant produced higher levels of NO}

Figure 4A shows the NO fluorescence signal at 120 min after HUVEC were exposed to placental explant supernatants (Control NT and Control PE). There was a significant difference between HUVEC treated with Control NT supernatant compared to cells exposed to Control PE supernatant, with higher NO production in Control NT supernatant-treated cells. Figure 4B-D shows NO generation by HUVEC after 60 min of incubation with DAF-FM ${ }^{\mathrm{TM}}$ after treatment with supernatant of placental explants. During this time of incubation (60 $\mathrm{min})$, a significantly higher NO production by HUVEC treated with Control NT supernatant compared to Control PE supernatant-treated cells as well as a high production of NO in VD PE supernatant treated cells compared to Control PE were observed (Figure 4B). When cultured with pretreated $\mathrm{H}_{2} \mathrm{O}_{2} \mathrm{NT}$ supernatant, the cells showed lower NO production (Figure $4 \mathrm{C}$ ). There was no significant difference between the production of $\mathrm{NO}$ in $\mathrm{H}_{2} \mathrm{O}_{2}$ supernatant treated cells compared to $\mathrm{H}_{2} \mathrm{O}_{2}+\mathrm{VD}$ (Figure 4D).

\section{Measurement of lipid peroxidation - TBARS}

The levels of MDA are shown in Figure 5A. Significantly higher MDA production was detected in HUVEC treated with Control PE explant supernatants compared to Control NT as well as compared to the VD $\mathrm{PE}$ group. MDA levels were significantly higher in the generated supernatant of HUVEC treated with $\mathrm{H}_{2} \mathrm{O}_{2}$ NT supernatant compared to Control NT and $\mathrm{H}_{2} \mathrm{O}_{2}+$ VD NT, as well as in the $\mathrm{H}_{2} \mathrm{O}_{2} \mathrm{PE}$-treated group compared to $\mathrm{H}_{2} \mathrm{O}_{2}+$ VD PE.
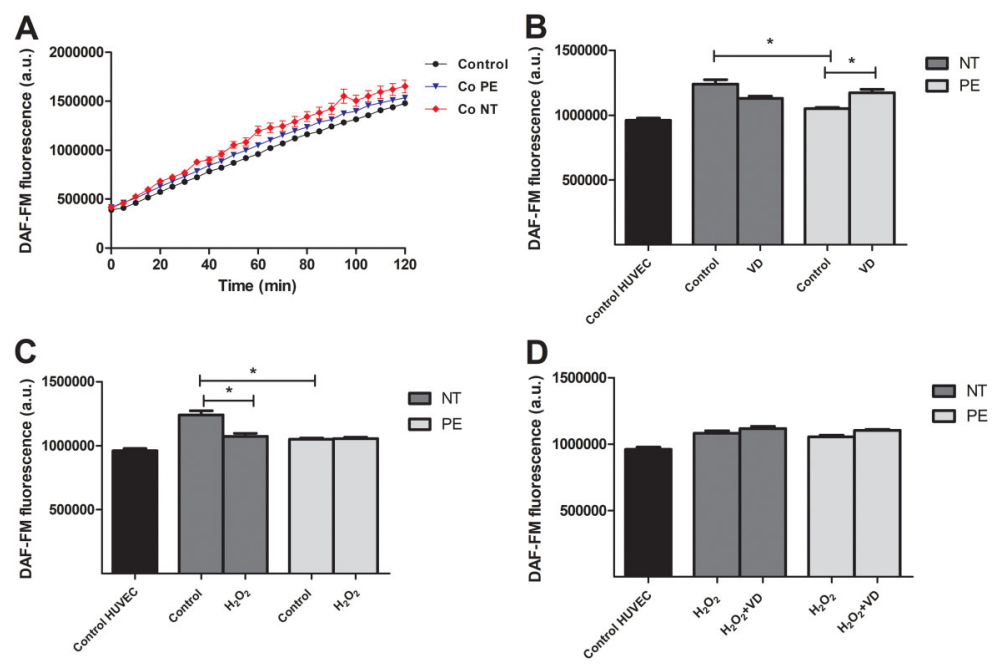

Figure 4. Nitric oxide fluorescence signal measured using DAF-FM ${ }^{\mathrm{TM}}$ after human umbilical vein endothelial cells (HUVEC) were exposed to placental explant supernatants from preeclamptic (PE) $(n=8)$ and normotensive (NT) pregnant women $(n=8)$. A, Control, Control NT, and Control PE; B, effect of treatment with vitamin D (VD) at $60 \mathrm{~min}$; C, effect of treatment with $\mathrm{H}_{2} \mathrm{O}_{2}$ at 60 min; and D, effect of treatment with $\mathrm{H}_{2} \mathrm{O}_{2}+\mathrm{VD}$ at 60 min. Data are reported as means $\pm \mathrm{SD}$ for one independent experiment, performed in quintuplicates. ${ }^{*} \mathrm{P}<0.05$ (ANOVA followed by Bonferroni's multiple comparison test). a.u.: arbitrary units. 

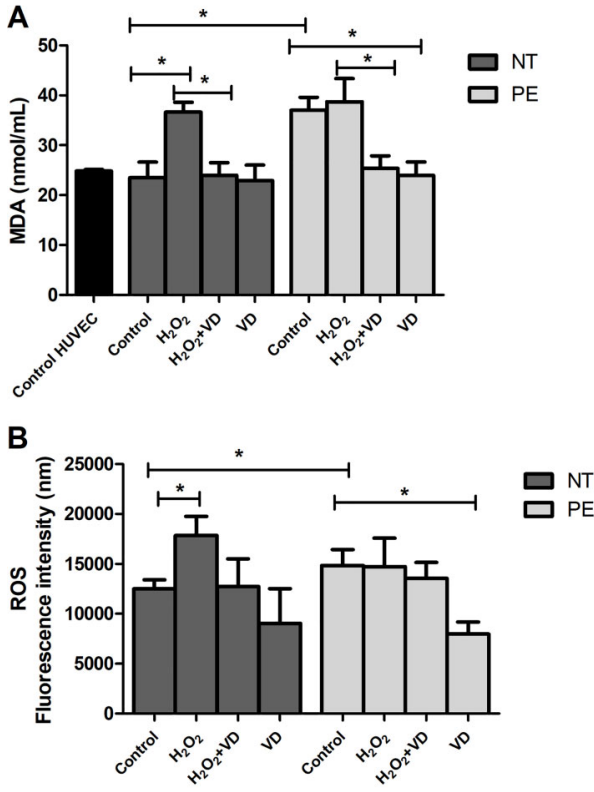

Figure 5. Malondialdehyde (MDA) levels (A) and levels of intracellular reactive oxygen species (ROS) (B) evaluated by fluorescence of 2,7-dichlorodihydrofluorescein diacetate (DCFH$D A$ ) in the supernatant of human umbilical vein endothelial cells (HUVEC) treated with supernatant of placental explants from preeclamptic $(\mathrm{PE})(\mathrm{n}=8)$ and normotensive $(\mathrm{NT})$ pregnant women $(n=8)$, cultured in the absence (Control) or presence of $\mathrm{H}_{2} \mathrm{O}_{2}$, $\mathrm{H}_{2} \mathrm{O}_{2}+$ vitamin $\mathrm{D}(\mathrm{VD})$, and VD. Data are reported as means \pm $\mathrm{SD}$. ${ }^{*} \mathrm{P}<0.05$ (ANOVA followed by Bonferroni's multiple comparison test).

Supernatants from PE explants cultured with vitamin $D$ decreased levels of intracellular ROS in HUVEC

Figure 5B shows the ROS levels in the groups. Oxidative stress was higher in HUVEC cultured with PE control supernatant compared to the NT one. Supernatants from NT placental explants cultured with $\mathrm{H}_{2} \mathrm{O}_{2}$ increased oxidative stress in HUVEC, while supernatants from $P E$ explants cultured with vitamin $D$ decreased oxidative stress in these cells.

\section{Discussion}

In the present study, we demonstrated that placental explants from PE women cultured without stimulus produced endogenous levels of the proinflammatory cytokines IL-1 $\beta$, TNF- $\alpha$, and IL-18. These results are in accordance with our previous studies showing a significant increase in NLRP3 inflammasome, caspase-1, IL-1 $\beta$, and TNF- $\alpha$ in placental villi and in placental homogenate from PE women compared to NT pregnant women (19) and suggested the involvement of the placenta in the exaggerated inflammatory state that characterizes PE. In addition, this inflammatory process and the intense oxidative stress originated in the placenta may give rise to endothelial dysfunction in PE (7). Therefore, we evaluated the effect of the supernatant from placental explants of PE and NT pregnant women treated with or without VD on oxidative stress and NO bioavailability in HUVEC. In our understanding, no studies have evaluated the effect of these supernatants on endothelial cells function. Thus, we studied placentas from NT pregnant women at term and placentas of PE women in the last trimester of pregnancy, at the time of delivery.

The present study demonstrated that HUVEC cultured with endogenous supernatants of non-treated placental explants from PE women produced increased levels of $\mathrm{NO}_{2}^{-}, \mathrm{NO}_{3}{ }^{-}, \mathrm{MDA}$, and ROS. These results suggest that the endogenous activation state of $\mathrm{PE}$ placental tissues triggered oxidative stress in these endothelial cells. In opposition, significantly higher production of NO was detected after HUVEC treatment with control supernatant of NT placental explants compared with the PE group, as well as after treatment with supernatant obtained from PE explants cultured with VD. Thus, these results demonstrated that HUVEC treated with supernatant from placental explants pre-cultured with VD showed a decrease in oxidative stress and an increase in the bioavailability of NO. This hypothesis may be raised since the concentration of IL- $1 \beta, \mathrm{TNF}-\alpha$, and IL-18 in the supernatant of placental explants from preeclamptic women decreased after treatment with VD, showing its modulatory effect on inflammatory cytokines production, particularly for its regulatory activities on the inflammatory response (20).

It has been proposed that the decrease in NO production in the placenta could cause abnormal tissue perfusion, which is observed in PE states. Regarding systemic production of $\mathrm{NO}$, it was shown that the vasodilation of the brachial artery mediated by NOdependent flow was approximately three times lower in pregnant women with $\mathrm{PE}$ compared to normal pregnant women (21). The NO system is also deranged in PE, as $\mathrm{NO}$ is a potent vasodilator, acting in order to induce relaxation in vascular smooth muscle cells (22). Decreased levels of NO have been reported in PE (23), and could be correlated with metabolic changes, such as hypertension, proteinuria, and platelet dysfunction (24).

Oxidative stress is an important phenomenon in PE (25) that could result from injury caused by hypoxia and reperfusion (26) and/or deficiency of antioxidant defenses (27). Peraçoli et al. (28) reported the endogenous activation of monocytes of pregnant women with $\mathrm{PE}$, demonstrated by correlation among high production of superoxide anion $\left(\mathrm{O}_{2}^{-}\right)$, hydrogen peroxide $\left(\mathrm{H}_{2} \mathrm{O}_{2}\right)$, and TNF- $\alpha$ by these cells, and high serum levels of uric acid, contributing to the enhanced oxidative and inflammatory state characteristic of PE. Hyperuricemia was also detected in the preeclamptic women in the present study, associated with high production of inflammatory cytokines by placental explants. According to other 
authors, production of uric acid concomitant with $\mathrm{O}_{2-}$ generation decreases $\mathrm{NO}$ bioavailability, leading to endothelial dysfunction (29). On the other hand, uric acid is also known as a powerful antioxidant through scavenging radical species and could also be regarded as a compensatory mechanism to counteract oxidative stress (29-31). Therefore, as hyperuricemia is a frequent finding in severe cases of PE, studies on oxidant and antioxidant properties of uric acid are of utmost importance to understand the pathophysiology of PE.

Several studies have been trying to show the effects of antioxidant therapy in women with PE. A systematic review performed by Rumbold et al. (32) concluded that supplementation with any antioxidant during pregnancy compared with control or placebo is associated with a reduced risk of developing PE. Similarly, Vadillo-Ortega et al. (33) showed that antioxidants in combination with $\mathrm{L}$-arginine are effective in cases of PE risk. Evidence of association between vitamin D status and PE has been shown in various studies, but clinical trials did not show an independent effect of supplementation in cases of PE prevention. However, problems regarding dose, timing, and duration of supplementation with VD have not been completely explored (34).

Some authors have already shown that normal pregnancy is associated with an increase in oxidative stress and lipid peroxidation, but antioxidants also increase (35). In opposition, women with PE present an insufficient production of antioxidants to offset the increase in oxidative stress and lipid peroxidation (36).

An important oxidative stress biomarker, MDA is a product of fatty acid oxidation and an indicator of lipid peroxidation. Some authors showed increased MDA levels in the blood of pregnant women with PE (37). MDA binds to TBARS, which are also elevated in the blood of women with PE (37), reflecting an oxidative stress status. In the present study, we observed a significant increase in MDA and intracellular ROS in HUVEC treated with supernatant control from placental explants of PE compared to NT. On the other hand, the

\section{References}

1. American College of Obstetricians and Gynecologists. Clinical Management Guidelines for Obstetrician-Gynecologists. Acog practice bulletin No. 202: Gestational hypertension and preeclampsia. Obstet Gynecol 2009; 133: e1-e25.

2. Folk DM. Hypertensive disorders of pregnancy: overview and current recommendations. J Midwifery Womens Health 2018; 63: 289-300, doi: 10.1111/jmwh.12725.

3. Moncada S, Higgs A. The L-arginine nitric-oxide pathway. N Eng J Med 1993; 329: 2002-2012, doi: 10.1056/NEJM1993 12303292706.

4. Krause BJ, Hanson MA, Casanello P. Role of nitric oxide in placental vascular development and function. treatment with VD supernatant decreased these levels, demonstrating the antioxidant and anti-inflammatory effect. Wimalawansa (38) highlighted the effects of VD as one of the key controllers of systemic inflammation and oxidative stress, downregulating oxidative stress, cell and tissue damage, and the aging process. In the same way, the hypovitaminosis is enhances oxidative stress and systemic inflammation. VD is also known as a potent anti-oxidant that facilitates balanced mitochondrial activities, preventing oxidative stress-related protein oxidation, lipid peroxidation, and DNA damage (38). Our findings are consistent with previous studies suggesting that the overproduction of free radicals and lipid peroxidation are important factors in the pathogenesis of preeclampsia (39). In summary, oxidative stress could contribute to the cytotoxic mechanisms in PE, inducing cellular damage, leading to endothelial cell injury, inflammation, and angiogenic imbalance (40).

In conclusion, our results suggest that placental explants of preeclamptic women treated with VD can decrease oxidative stress and lipid peroxidation, as well as increase the bioavailability of NO in HUVEC.

\section{Supplementary Material}

Click to view [pdf].

\section{Acknowledgments}

The authors would like to thank Carina Nihlén and Annika Olsson, from the Department of Physiology and Pharmacology at Karolinska Institutet for their expertise in the HPLC technique, important methodology in the development of this work. This work was supported by the São Paulo Research Foundation (FAPESP-Brazil) (grant numbers 2016/23452-2 and 2019/00234-8), Swedish Research Council (grant numbers: 2016-01381 and 2020-01645), Swedish Heart and Lung Foundation (grant numbers: 20170124 and 20180568), and NovoNordisk (grant number: 2019\#0055026).
Placenta 2011; 32: 797-805, doi: 10.1016/j.placenta. 2011.06.025.

5. Poniedzialek-Czajkowska E, Marciniak B, Kimber-Trojnar Z, Leszczyńska-Gorzelak B, Oleszczuket J. Nitric oxide in normal and preeclamptic pregnancy. Curr Pharm Biotechnol 2011; 12: 743-749, doi: 10.2174/13892011179547 0921.

6. Farrow KN, Lakshminrusimha S, Reda WJ, Wedgwood S, Czech L, Gugino SF, et al. Superoxide dismutase restores eNOS expression and function in resistance pulmonary arteries from neonatal lambs with persistent pulmonary hypertension. Am J Physiol Lung Cell Mol Physiol 2008; 295: L979-L987, doi: 10.1152/ajplung.90238.2008. 
7. Cindrova-Davies T. Gabor than Award Lecture: pre-eclampsia - from placental oxidative stress to maternal endothelial dysfunction. Placenta 2009; 30: S55-S65, doi: 10.1016/j. placenta.2008.11.020.

8. Mittal M, Siddiqui MR, Tran K, Reddy SP, Malik AB. reactive oxygen species in inflammation and tissue injury. Antioxid Redox Signal 2014; 20: 1126-1167, doi: 10.1089/ars.2012. 5149.

9. Xu L, Lee M, Jeyabalan A, Roberts JM. The relationship of hypovitaminosis D and IL-6 in preeclampsia. Am J Obstet Gynecol 2014; 210: 149.e1-7, doi: 10.1016/j.ajog.2013.09. 037.

10. Murthi P, Yong HEJ, Ngyuen TPH, Ellery S, Singh H, Rahman $\mathrm{R}$, et al. Role of the placental vitamin $\mathrm{D}$ receptor in modulating feto-placental growth in fetal growth restriction and preeclampsia-affected pregnancies. Front Physiol 2016; 7: 43, doi: 10.3389/fphys.2016.00043.

11. Robinson CJ, Wagner CL, Hollis BW, Baatz JE, Johnson DD. Association of maternal vitamin $D$ and placenta growth factor with the diagnosis of early onset severe preeclampsia. Am J Perinatol 2013; 30: 167-172.

12. Mohaghegh Z, Abedi P, Dilgouni T, Namvar F, Ruzafza S. The relation of preeclampsia and serum level of 25hydroxyvitamin $D$ in mothers and their neonates: a case control study in iran. Horm Metab Res 2015; 47: 284-288, doi: 10.1055/s-0034-1395607.

13. Hyppönen E, Cavadino A, Williams D, Fraser A, Vereczkey $A$, Fraser WD, et al. Vitamin $D$ and pre-eclampsia: original data, systematic review and meta-analysis. Ann Nutr Metab 2013; 63: 331-340, doi: 10.1159/000358338.

14. Nunes PR, Peracoli MTS, Romao-Veiga M, Matias ML, Ribeiro VR, Fernandes CJC, et al. Hydrogen peroxidemediated oxidative stress induces inflammasome activation in term human placental explants. Pregnancy Hypertens 2018; 14: 29-36, doi: 10.1016/j.preghy.2018.07.006.

15. Matsubara K, Higaki T, Matsubara Y, Nawa A. Nitric oxide and reactive oxygen species in the pathogenesis of preeclampsia. Int J Mol Sci 2015; 16: 4600-4614, doi: 10.3390/ijms 16034600.

16. Bischoff-Ferrari HA, Giovannucci E, Willet WC, Dietrich T, Dawson-Hughes B. Estimation of optimal serum concentrations of 25-hydroxyvitamin $D$ for multiple health outcomes. Am J Clin Nutr 2006; 84: 18-28, doi: 10.1093/ajcn/84.1.18.

17. Larionov A, Krause A, Miller W. A standard curve based method for relative real-time PCR data processing. BMC Bioinformatics 2005; 6: 62, doi: 10.1186/1471-2105-6-62.

18. Montenegro MF, Sundqvist $M L$, Nihlén $C$, Hezel $M$, Carlström M, Weitzberg E, et al. Profound differences between humans and rodents in the ability to concentrate salivary nitrate: Implications for translational research. Redox Biol 2016; 10: 206-210, doi: 10.1016/j.redox.2016.10.011.

19. Weel IC, Romão-Veiga M, Matias ML, Fioratti EG, Peraçoli $\mathrm{JC}$, Borges VT, et al. Increased expression of NLRP3 inflammasome in placentas from pregnant women with severe preeclampsia. J Reprod Immunol 2017; 123: 40-47, doi: 10.1016/j.jri.2017.09.002.

20. Prietl B, Treiber G, Pieber TR, Amrein K. Vitamin D and immune function. Nutrients 2013; 5: 2502-2521, doi: 10.3390/nu5072502.
21. Cockell AP, Poston L. Flow-mediated vasodilatation is enhanced in normal pregnancy but reduced in preeclampsia. Hypertension 1997; 30: 247-251, doi: 10.1161/01. HYP.30.2.247.

22. Quillon A, Fromy B, Debret R. Endothelium microenvironment sensing leading to nitric oxidemediated vasodilation: a review of nervous and biomechanical signals. Nitric Oxide 2015; 45: 20-26, doi: 10.1016/j.niox.2015.01.006.

23. Sankaralingam S, Xu H, Davidge ST. Arginase contributes to endothelial cell oxidative stress in response to plasma from women with preeclampsia. Cardiovasc Res 2010; 85: 194-203, doi: 10.1093/cvr/cvp277.

24. Kulandavelu S, Whiteley KJ, Qu D, Mu J, Bainbridge SA, Adamson SL. Endothelial nitric oxide synthase deficiency reduces uterine blood flow, spiral artery elongation, and placentaloxygenation in pregnant mice. Hypertension 2012; 60: 231-238, doi: 10.1161/HYPERTENSIONAHA. 111.187559.

25. Burton GJ, Jauniaux E. Placental oxidative stress: from miscarriage to preeclampsia. J Soc Gynecol Investig 2004; 11: 342-352, doi: 10.1016/j.jsgi.2004.03.003.

26. Burton GJ, Hung TH. Hypoxia-reoxygenation: a potential source of placental oxidative stress in normal pregnancy and preeclampsia. Fetal Mat Med Rev 2003; 14: 97-117, doi: 10.1017/S0965539503001049.

27. Perkins AV. Endogenous anti-oxidants in pregnancy and preeclampsia. Aust N Z J Obstet Gynaecol 2006; 46: 77-83, doi: 10.1111/j.1479-828X.2006.00532.x.

28. Peraçoli MTS, Bannwart CF, Cristofalo R, Borges VTM, Costa RAA, Witkin SS, et al. Increased reactive oxygen species and tumor necrosis factor-alpha production by monocytes are associated with elevated levels of uric acid in pre-eclamptic women. Am J Reprod Immunol 2011; 66: 460-467, doi: 10.1111/j.1600-0897.2011.01016.x.

29. Maruhashi T, Hisatome I, Kihara Y, Higashi Y. Hyperuricemia and endothelial function: From molecular background to clinical perspectives. Atherosclerosis 2018; 278: 226-231, doi: 10.1016/j.atherosclerosis.2018.10.007.

30. Ames BN, Cathcart R, Schwiers E, Hochstein P. Uric acid Uric acid provides an antioxidant defense in humans against oxidant- and radical-caused aging and cancer: a hypothesis. Proc Natl Acad Sci USA 1981; 78: 6858-6862, doi: 10.1073/ pnas.78.11.6858.

31. Martin AC, Brown MA. Could uric acid have a pathogenic role in pre-eclampsia? Nat Rev Nephrol 2010; 6: 744-748, doi: 10.1038/nrneph.2010.125.

32. Rumbold A, Duley L, Crowther CA, Haslam RR. Antioxidants for preventing pre-eclampsia. Cochrane Database Syst Rev 2008; 2008: CD004227.

33. Vadillo-Ortega F, Perichart-Perera O, Salvador E, AvilaVergara MA, Ibarra I, Ahued R, et al. Effect of supplementation during pregnancy with L-arginine and antioxidant vitamins in medical food on pre-eclampsia in high risk population: randomised controlled trial. BMJ 2011; 342: d2901, doi: 10.1136/bmj.d2901.

34. Purswani JM, Gala P, Dwarkanath P, Larkin HM, Kurpad A, Saurabh M. The role of vitamin D in pre-eclampsia: a systematic review. BMC Pregnancy Childbirth 2017; 17: 231, doi: 10.1186/s12884-017-1408-3. 
35. Sekiba K, Yoshioka T. Changes of lipid peroxidation, and superoxide dismutase activity in the human placenta. $\mathrm{Am} \mathrm{J}$ Obstet Gynecol 1979; 135: 368-371, doi: 10.1016/00029378(79)90707-5.

36. Davidge ST, Hubel CA, Brayden RD, Capeless EC, McLaughlin MK. Sera antioxidant activity in uncomplicated and preeclamptic pregnancies. Obstet Gynecol 1992; 79: 897-901.

37. Madazli R, Benian A, Aydin S, Uzun H, Tolun N. The plasma and placental levels of malondialdehyde, glutathione, and superoxide dismutase in preeclampsia. Obstet Gynaecol 2002; 22: 477-480, doi: 10.1080/0144361021000003573.
38. Wimalawansa SJ. Vitamin D deficiency: effects on oxidative stress, epigenetics, gene regulation, and aging. Biology (Basel) 2019; 8: 30.

39. Padmini E, Lavanya S, Uthra V. Preeclamptic placental stress and over expression of mitochondrial HSP70. Clin Chem Lab Med 2009; 47: 1073-1080, doi: 10.1515/ CCLM.2009.247.

40. Chiarello DI, Abad C, Rojas D, Toledo F, Vázquez CM, Mate $\mathrm{A}$, et al. Oxidative stress: normal pregnancy versus preeclampsia. Biophys Acta Mol Basis Dis 2020; 1866: 165354, doi: 10.1016/j.bbadis.2018.12.005. 